\title{
In situ Fabrication of 3-Dimensional Chemical Patterns in Fused Silica Separation Capillaries with Polymerized Phospholipids
}

\author{
Eric E. Ross, Elisabeth Mansfield, Yiding Huang and Craig A. Aspinwall* \\ Department of Chemistry, University of Arizona, Tucson, AZ 85721
}

Supporting Information

\section{Experimental}

Fused silica capillaries purchased from Polymicro Technologies, LLC (Phoenix, AZ) were used in all experiments. The polyimide coating was removed from areas of the capillary that served as windows through which polymerization visualization was performed. Prior to deposition of the lipid bilayer, the interior of the fused silica capillaries was cleaned with piranha solution (70\% sulfuric acid/30\% hydrogen peroxide (30\%)) for 30 minutes followed by repetitive rinses with deionized water.

All lipid solutions were prepared by delivering aliquots from stock lipid solutions in chloroform or benzene (bis-SorbPC only) to small vials and drying with a direct stream of argon gas prior to vacuum desiccation for greater than 4 hours. The dried lipid films were rehydrated in water or $\mathrm{pH}=7.4$ buffer to a concentration of $1 \mathrm{mg} / \mathrm{mL}$ and sonicated to clarity to produce small unilamellar vesicles approximately $50-70 \mathrm{~nm}$ in size. The resultant lipid vesicle suspensions were injected into the capillary for 30 minutes. Excess lipid and non-deposited lipid vesicles were removed by rinses with water or buffer.

Polymerization of bis-SorbPC coated capillaries was performed using direct UV polymerization by exposing the lipid bilayer coated fused silica capillaries to light from a low-pressure mercury pen lamp (UVP, Inc) with a rated intensity of 4500 $\mu \mathrm{W} / \mathrm{cm}^{2}$ at $254 \mathrm{~nm}$. The polymerizable lipid monomers used in this work have an absorbance maximum at $\sim 260 \mathrm{~nm}$. Variable feature dimension photomasks were constructed in-house using either a) narrow strips of opaque tape or b) $150 \mu \mathrm{m}$ o.d. opaque capillaries assembled onto a fused silica microscope slide. The mask was placed directly onto the lipid bilayer containing capillary. The UV source was placed $10 \mathrm{~cm}$ from the capillary and used to irradiate the capillary for 10 minutes. Unpolymerized lipid was removed with $1 \%$ (v/v) Triton X-100 followed by passage of $\mathrm{N}_{2}$ or Ar gas through the capillary. The rinse procedure was repeated 2-5 times to ensure complete removal of unpolymerized lipid. The capillary was rinsed extensively with deionized water prior to utilization and/or storage.

Functionalization of capillaries was performed by deposition of mixed lipid vesicles containing 90-95\% dioleylphosphatidylcholine (DOPC) and 5-10\% headgroup functionalized phospholipids as described in text. Following vesicle fusion and subsequent assembly of the functionalized bilayer into the bare regions of the capillary, excess vesicles were removed via a series of buffer rinses.

Fluorescent images were collected on a Nikon Eclipse Quantum TE2000 with a 4x/0.13 N.A. objective and a Cascade 650 front illuminated CCD camera (Roper Scientific, Tucson, AZ). MetaVue 5.0 software was used to capture and analyze all images(Universal Imaging Corp.). 